OPEN

SUBJECT AREAS: GENETIC VECTORS

COCHLEA

Received

25 October 2013

Accepted

1 April 2014

Published

22 April 2014

Correspondence and requests for materials should be addressed to

R.T.R. (rrichardson@

bionicsinstitute.org)

\section{Viability of Long-Term Gene Therapy in} the Cochlea

\author{
Patrick J. Atkinson ${ }^{1,2}$, Andrew K. Wise ${ }^{1,2,4}$, Brianna O. Flynn' ${ }^{1}$, Bryony A. Nayagam ${ }^{1,3}$ \\ \& Rachael T. Richardson ${ }^{1,2,4}$
}

\author{
'Bionics Institute, East Melbourne, Victoria, Australia, ${ }^{2}$ Department of Otolaryngology, University of Melbourne, East Melbourne, \\ Victoria, Australia, ${ }^{3}$ Department of Audiology and Speech Pathology, University of Melbourne, Parkville, Victoria, Australia, \\ ${ }^{4}$ Department of Medical Bionics, University of Melbourne, East Melbourne, Victoria, Australia.
}

Gene therapy has been investigated as a way to introduce a variety of genes to treat neurological disorders. An important clinical consideration is its long-term effectiveness. This research aims to study the long-term expression and effectiveness of gene therapy in promoting spiral ganglion neuron survival after deafness. Adenoviral vectors modified to express brain derived neurotrophic factor or neurotrophin-3 were unilaterally injected into the guinea pig cochlea one week post ototoxic deafening. After six months, persistence of gene expression and significantly greater neuronal survival in neurotrophin-treated cochleae compared to the contralateral cochleae were observed. The long-term gene expression observed indicates that gene therapy is potentially viable; however the degeneration of the transduced cells as a result of the original ototoxic insult may limit clinical effectiveness. With further research aimed at transducing stable cochlear cells, gene therapy may be an efficacious way to introduce neurotrophins to promote neuronal survival after hearing loss.
C onventional pharmalogical and surgical interventions are currently ineffective or unavailable for the treatment of a number of diseases within the central and peripheral nervous systems. As such, more novel approaches are currently being examined. One of these approaches is the use of gene therapy to restore function, prevent degeneration or even replace lost cells. Gene therapy has been utilised in a number of conditions at both the pre-clinical and clinical stage; including Parkinson's disease ${ }^{1,2}$, retinal blindness ${ }^{3,4}$ and hearing $\operatorname{loss}^{5-8}$. Gene therapy treatments for Parkinson's disease and various forms of retinal blindness have shown great promise, with transgene expression persisting for up to six years in a non-human primate model of Parkinson's disease? and with many studies reaching phase II clinical trials. Whilst the use of gene therapy in animal models of hearing loss has also yielded positive results, there remain questions surrounding the longevity of gene expression within the cochlea, its long-term efficacy and its safety ${ }^{7,8,10}$.

The deaf cochlea provides a model to study gene therapy in the context of neural and tissue degeneration. After hearing loss induced by ototoxocity, for example, the sensory hair cells die, stimulating spiral ganglion neuron (SGN) degeneration and degeneration of the organ of Corti (OC). Gene therapy has been studied as a means to prevent the neural degeneration after hearing loss in order to preserve the neural elements required for cochlear implant use. Neurotrophin (NT) gene therapy that was targeted to the OC was shown to be more efficacious for preventing the degeneration of SGNs compared to gene expression that was expressed broadly throughout the cochlea ${ }^{7}$. However, the ototoxically-induced degeneration of the OC continued, even after early intervention with NT-gene therapy ${ }^{10,11}$. Despite this, gene expression has been observed for up to 11 weeks in the OC of the deafened cochlea with the NT gene expression resulting in SGN survival compared to contralateral cochleae ${ }^{10}$. However, from the time points thus far examined it could not be determined whether this degeneration would result in transduced cells undergoing apoptosis, thereby limiting gene expression and thus neurotrophic support for SGNs.

This paper will further elucidate the longevity and efficacy of adenovirus (Ad) which has been modified to express green fluorescent protein (GFP) in combination with either brain derived neurotrophic factor (BDNF) or neurotrophin-3 (NT3) (hereon in referred to as Ad-NTs) in order to help establish the suitability of NT gene therapy for maintaining SGNs after hearing loss. This will be achieved by examining the extent of OC degeneration, the longevity and pattern of gene expression, SGN density and peripheral fibre density, in ototoxically deafened guinea pig (GP) cochleae six months post-NT-gene therapy, a time point at which very few SGNs remain after deafness in the untreated $\mathrm{GP}^{12}$. 

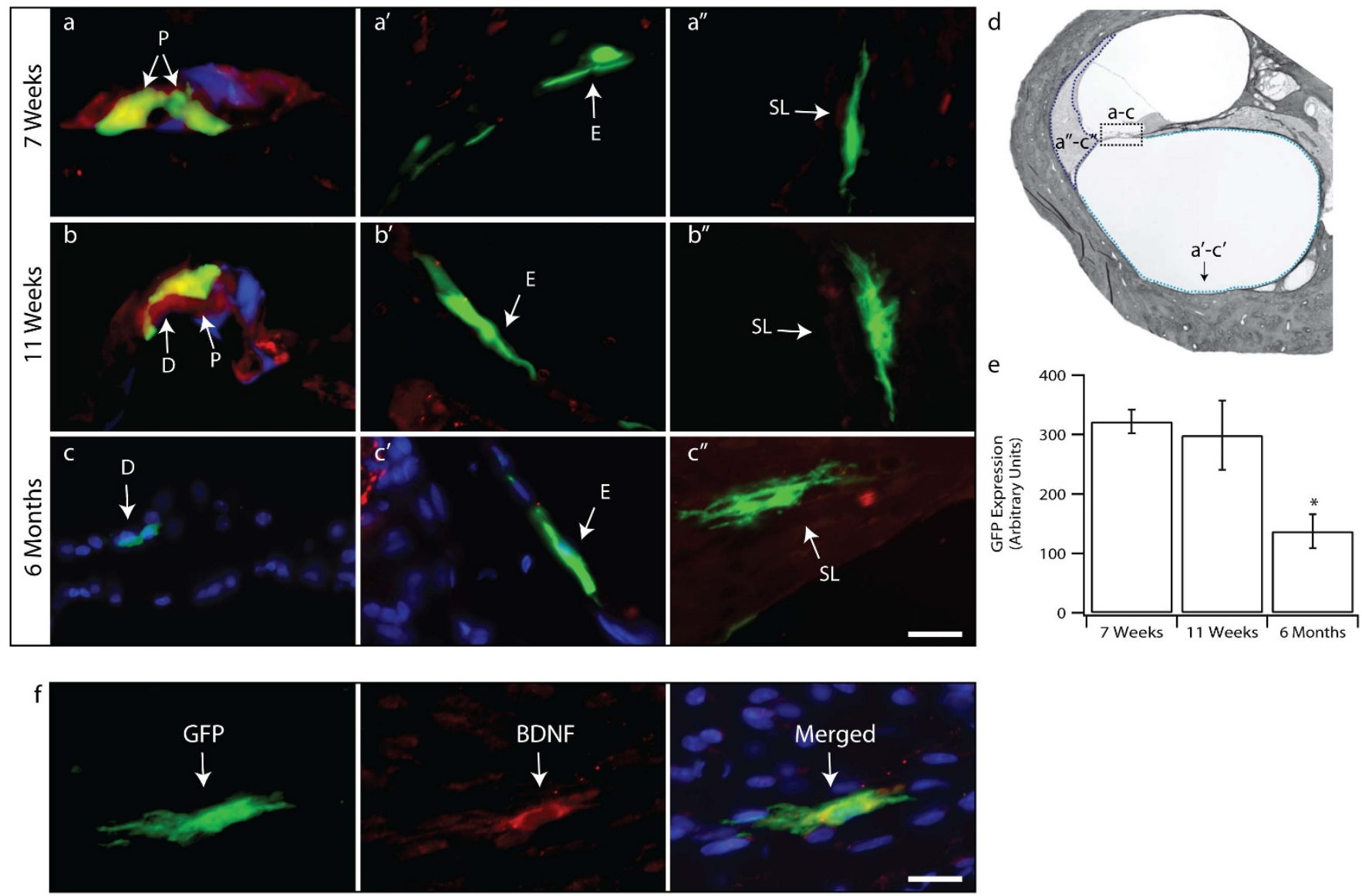

Figure 1 Viral expression in the deafened cochlea 7 weeks $^{10}(\mathrm{a}), 11$ weeks ${ }^{10}$ (b) or six months (c) post injection. (a-c) GFP expression $($ green) within the degenerated OC. Transduced cells included the pillar cells (P) and Deiters' cells (D). GFP was also detected in non-OC cells namely in the endosteal cells $\left(E ; a^{\prime}-c^{\prime}\right)$ and the cells of the spiral limbus (SL; $\left.\mathrm{a}^{\prime \prime}-\mathrm{c}^{\prime \prime}\right)$. The location of these cells is annotated on a histological mid-modiolar section of the cochlear lower basal turn (d). The level of expression was significantly lower after 6 months compared to 7 or 11 weeks of treatment, as determined by the density of GFP-positive pixels in mid-modiolar cochlear sections (e; ${ }^{*} \mathrm{p}<0.05$, ANOVA). (f) NT expression was confirmed by co-localisation of GFP (green) with BDNF (red). Scale bar for all images $=25 \mu \mathrm{m}$. $(\mathrm{a}-\mathrm{b})$ red $=$ calretinin, blue $=$ phalloidin; $(\mathrm{c})$ red $=$ calretinin, blue $=$ DAPI; $(\mathrm{f})$ blue $=$ DAPI .

\section{Results}

Viral gene expression profile. The GFP reporter gene present in AdNTs was used to examine the gene expression pattern within the ototoxically deafened cochlea six months post-injection. For the first time, these results show that viral vector expression is present for at least six months after inoculation in the deafened GP cochlea, with NT expression confirmed by co-localisation of GFP and BDNF. However, the overall GFP expression was significantly lower at 6 months compared to the shorter time-points examined in previous studies, as calculated by the density of GFP-positive pixels in midmodiolar cochlear sections ( $p<0.05$, ANOVA; figure 1$)^{7,10,13}$. Gene expression was predominately restricted to the basal turn of the cochlea, proximal to the site of injection. Gene expression beyond this area was observed in 1 GP only, in which GFP expression was observed in the lower middle turn of the cochlea. These results are consistent with previous studies ${ }^{7,8,10}$. The cells transduced within these regions included the pillar cells and Deiters' cells of the OC, cells of the spiral ligament and endosteal cells lining the endolymphatic and perilymphatic spaces. Transduced cell types in these GPs were consistent with those previously observed after 7 or 11 weeks of treatment (figure 1).

Concomitantly, when examined six months post-deafening there was minimal calretinin positive staining indicating a loss of $\mathrm{HCs}$ and degeneration of some supporting cells of the OC. Morphological analysis with $\mathrm{H}+\mathrm{E}$ staining confirmed a flattening of the OC, which is a pathological response to ototoxic deafening ${ }^{14}$ (figure 2). There was symmetrical degeneration of the OC observed between the left treated cochleae and the right non-treated cochleae (figure 2).

Effects of long-term of neurotrophin gene therapy on SGN survival. To test if a single viral injection of Ad-NTs is able to provide long-term protection of SGNs after deafness, the density of SGN cell bodies was examined in the basal, middle and apical turns 6 months post NT-gene therapy treatment. These densities were then compared to the contralateral non-injected cochleae (figure 3).

There was a significantly greater density of SGNs in the basal turn of GPs treated with Ad-NTs when compared to the non-treated contralateral cochleae $\left(895 \pm 87 \mathrm{SGN} / \mathrm{mm}^{2}\right.$ vs. $632 \pm 74 \mathrm{SGN} /$ $\mathrm{mm}^{2} ; \mathrm{p}<0.05$, paired t-test), indicating that NT-gene therapy provided significant nerve survival in the basal region. There were no differences in SGN densities between treated cochleae and the contralateral cochleae in the middle turn $\left(849 \pm 182 \mathrm{SGN} / \mathrm{mm}^{2}\right.$ vs. $596 \pm 70 \mathrm{SGN} / \mathrm{mm}^{2}$; p $>0.05$, paired t-test) or for the apical $\operatorname{turn}\left(533 \pm 130 \mathrm{SGN} / \mathrm{mm}^{2}\right.$ vs. $415 \pm 89 \mathrm{SGN} / \mathrm{mm}^{2} ; \mathrm{p}>0.05$, paired t-test). The overall survival observed was lower in this six month group compared to the 11 week data as denoted by the grey dashed lines on figure 3. In GPs treated with Ad modified to express GFP alone, there was no gain or loss of SGNs in cochleae injected with AdGFP compared to contralateral cochleae at 11 weeks post-injection ${ }^{10}$.

To determine the effect of NT-gene therapy on the peripheral fibres of SGNs in the deafened GP cochlea, the fibres were examined in cross-section of the OSL and compared to earlier time points. 
(a)

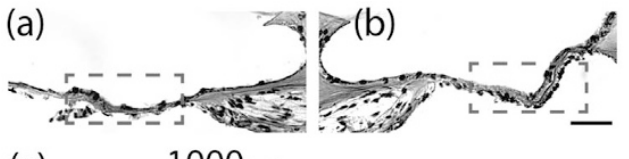

(c)

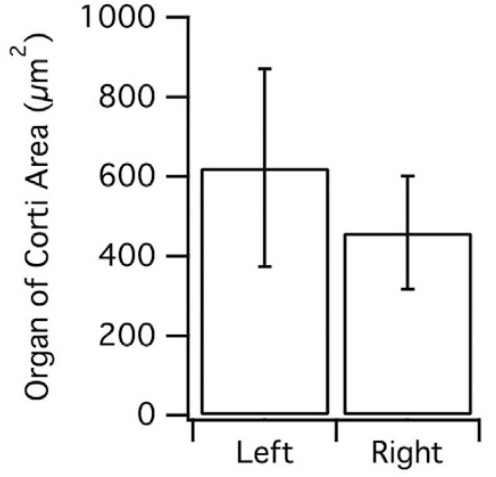

Figure $2 \mid$ OC degeneration six most post deafening. $\mathrm{H}+\mathrm{E}$ stained cross sections of the OC of the (a) left and the (b) right cochlea. (c) There was no difference observed in area of the OC between the left or right cochlea after systemic aminoglycoside-induced sensorineural hearing loss when measured six months post deafening.

There was significant SGN peripheral fibre preservation in the NTtreated cochleae compared to the contralateral non-treated cochlea when examined after seven weeks of treatment $(\mathrm{p}<0.001$, paired $\mathrm{t}-$ test; figure 4), however, no significant difference was observed after either 11 weeks or six months of NT-gene therapy, indicating that long term NT-gene therapy is unable to prevent the degeneration of peripheral fibres.

Safety and viability of long-term gene therapy in the deaf cochlea. Similar to previous findings ${ }^{10}$, there was no evidence of cochlear infiltration by multinucleated giant cells or macrophages following Ad-delivery into the scala media, demonstrating that after an extended period in the cochlea adenoviral based gene therapy does not illicit an immune response. When examined after six months there was only one cochlea in which a tissue response was observed. The mild tissue response observed was composed of fibrous tissue and new bone growth, and was localised to the scala tympani of the lower basal turn (figure 5). The area of the scala tympani occupied by the tissue response was measured as $19.7 \%$ in this particular case and no response was observed in the scala media or in any of the other turns of the cochlea.

\section{Discussion}

This study has demonstrated that Ad-mediated gene expression, as measured by both GFP expression and NT expression, is able to persist for up to six months in the cochlea post deafening, although gene expression was significantly reduced compared to earlier time points as demonstrated in figure 1. This is thought to be due to the continued degeneration of the OC that occurs after ototoxic injury ${ }^{8,10,14}$. Interestingly, NTs such as NT3, glial derived neurotrophic factor, and to a lesser extent BDNF, have been previously shown to play a protective role in the OC, when delivered prior to ototoxic insult $^{15,16}$. These findings, along with the results of the current study, suggest that whilst it is possible to use NTs to provide protection to the $\mathrm{OC}$ in some conditions, it may not be possible to do so using NT gene therapy after a severe ototoxic insult.

The lower level of GFP expression and the continued degeneration of the OC suggest that expression may become further diminished to the point that it is no longer observed in the OC at periods greater than six months post-inoculation into the scala media of ototoxically deafened cochlea. Importantly, despite the lowered gene expression in the OC after six months there was still expression in other cochlear areas such as endosteal cells ( $4 / 5$ of cochleae), interdental cells ( $2 / 5$ of cochleae) and SL (2/5 of cochleae), indicating that Ad-mediated expression is able to persist long term as long as the transduced cells remain viable. Therefore, for long-term gene expression it is necessary to target cell populations within the cochlea that do not degenerate after hearing loss. When viewed in the context of lowered viral expression (especially within the OC) the results suggest that, whilst the long-term survival effect is maintained, SGNs are degenerating as a result of the lower gene expression in the six month cohort. As such, NT-gene therapy targeting the OC may not be a viable way to maintain SGNs in the ototoxically deafened GP long-term and, therefore other cell populations should be considered for transduction. One possible population could be the spiral ganglion Schwann cells, which could be targeted through the use of a Schwann cell-specific promoter such as $\mathrm{PMP} 22^{17}$ and a surgical approach targeting this sub-population of cells.

When examined six months after inoculation, SGN survival was greater in the Ad-NTs treated cochlea compared to their contralateral cochlea, however the overall level of survival was decreased from earlier time points. This decrease in the level of survival correlated to a decrease in the level of gene expression. In the wider context though, gene therapy that is targeted to the OC should not be disregarded for several reasons. Firstly, the degeneration of the OC in the GP cochlea after ototoxic deafening progresses much faster than is observed in humans, and as such six months of deafness may

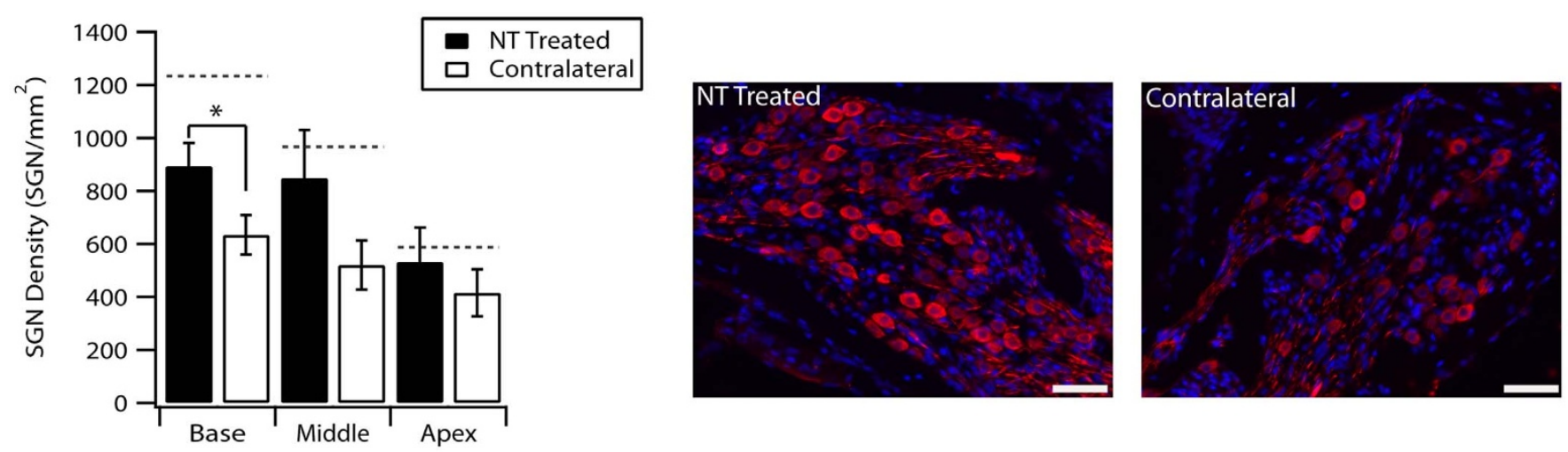

Figure 3 SGN density measurements in NT treated and contralateral cochleae of deafened GPs. When examined after six months of treatment there was a significantly greater density of SGNs in the basal turn of GPs treated with Ad-NTs compared to contralateral cochleae $\left({ }^{*} \mathrm{p}<0.05\right.$, paired t-test). Grey dashed lines indicate SGN survival after 11 weeks of Ad-NTs treatment reported in a previous study ${ }^{10}$. Example photomicrographs of SGNs $($ red $=$ NF-H and shows SGN cell bodies, blue $=$ DAPI) in the lower basal turn are shown. Error bars indicate the SEM $(n=5$ GPs per point). Scale bar $=50 \mu \mathrm{m}$. 

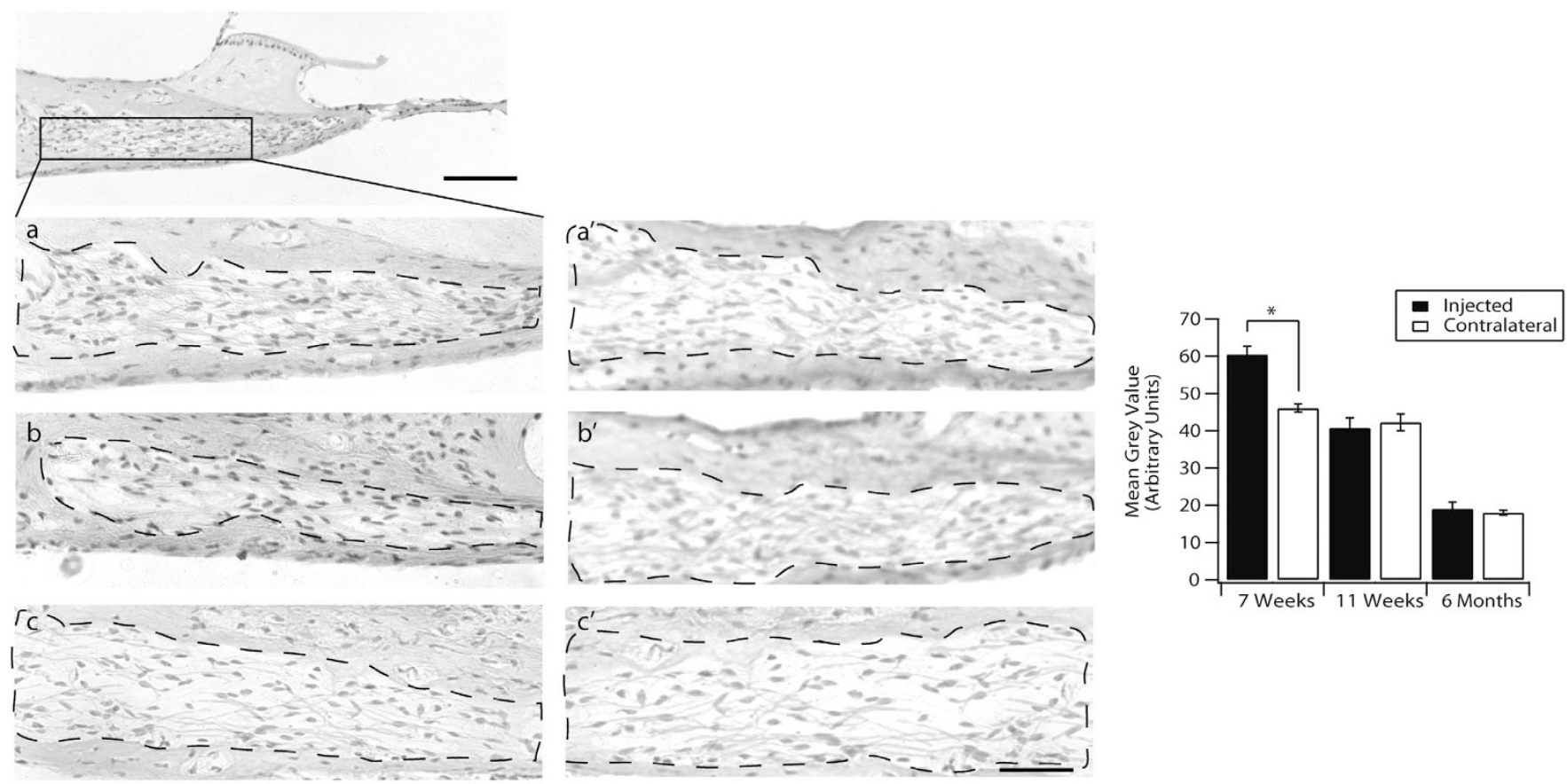

Figure $4 \mid$ Peripheral fibres in the deafened NTs-treated (a-c) and the contralateral $\left(a^{\prime}-c^{\prime}\right)$ GP cochlea as viewed in cross-section through the OSL. Peripheral fibres in the OSL after (a) 7 weeks, (b) 11 weeks and (c) six months of treatment with Ad-NTs. There was a significantly greater mean grey value measured in the OSL of the injected GP compared to the contralateral cochlea after 7 weeks $\left({ }^{*} \mathrm{p}<0.01\right.$; paired t-test). No difference between the treated and contralateral cochleae was observed after 11 weeks or six months. Scale bar $=100 \mu \mathrm{m}$ and $50 \mu \mathrm{m}$ in magnified image.

equate to many years or even decades in humans ${ }^{18-20}$. Secondly, not all forms of deafness result in the complete degeneration of the OC (e.g. noise induced hearing loss) and therefore gene therapy targeting the OC in this form of deafness could be clinically viable. Thirdly, while various other methods have been used experimentally to introduce NTs into the deafened cochlea with varying degrees of success, their ability to provide long-term support in ototoxically deafened GP has yet to be shown ${ }^{21-24}$. Neurotrophin delivery using miniosmotic pumps, for example, has been shown to be very effective during the treatment period, but this efficacy has not been shown to last more than two weeks after the cessation of treatment ${ }^{21,22}$. In contrast, gene therapy is a single-intervention technique that

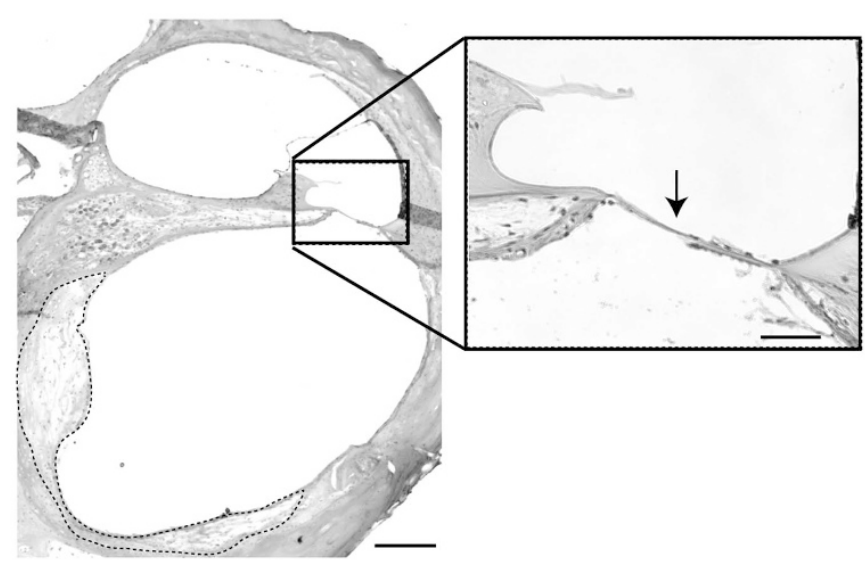

Figure $5 \mid$ Histological mid-modiolar example of tissue response six months post-viral injection. The mild tissue response (dotted line), characterised by fibrous tissue and new bone growth, was localised to the lower basal scala tympani with no response observed in the scala media (box). The degeneration of the OC (arrow) can also be observed in the magnified image. Scale bar $=200 \mu \mathrm{m}$ and $50 \mu \mathrm{m}$ in magnified image. provides long-term exposure to neurotrophins and has the potential to maintain SGNs long-term after hearing loss.

The findings presented in this study extend upon previous work and confirm several key results which are critical in determining the viability of NT-gene therapy. These include the ability of Ad mediated gene expression to persist for an extended period in the deafened cochlea, the ability to provide long-term support for neurons and the ability to do this safely without causing a significant immune response in the cochlea. The minimal tissue response observed could be further mitigated in a number of ways including the administration of the glucocorticoid steroid dexamethasone, which has been shown to reduce the immune response associated with insertion of a cochlear implant into the cochlea ${ }^{25}$, or through the use of a soft surgical approach such as entering through the round window rather than performing a cochleostomy ${ }^{26}$. In clinical cases, accessing the scala media through a round window approach is possible, but not trivial, and would involve gaining access to the middle ear by entering the cochlea between the facial nerve and the chorda tympani.

Whilst there remain technical challenges in bringing viral gene therapy to the clinic, this study has highlighted that gene therapy that is targeted to the organ of Corti of the cochlea is ideal for targeting the sensory cells but has limitations relating to the degeneration of the sensory cells after severe hearing loss. In future studies, targeting NT-gene therapy to cell populations that do not undergo degeneration such as the spiral ganglion Schwann cells may enable longer-term expression and in turn promote greater survival of SGNs and their peripheral fibres.

\section{Methods}

Ad vectors. Adenoviral vectors were generated as previously described ${ }^{7}$. Briefly, replication deficient adenovirus type 5 was genetically modified to expression GFP in concert with mouse BDNF or mouse NT3. Ad vectors were diluted $1: 5$ in artificial endolymph ( $120 \mathrm{mmol} / \mathrm{l} \mathrm{KCL}, 2.5 \mathrm{mmol} / \mathrm{l} \mathrm{NaCl}, 0.5 \mathrm{mmol} / \mathrm{l} \mathrm{MgCl}_{2}, 028 \mathrm{mmol} / \mathrm{l}$ $\left.\mathrm{CaCl}_{2}, 7.6 \mathrm{mmol} / \mathrm{l} \mathrm{K}_{2} \mathrm{HPO}_{4}, 2.7 \mathrm{mmol} / \mathrm{KH}_{2} \mathrm{PO}_{4}, \mathrm{pH} 7.4\right)$ to final concentrations of $3.0 \times 10^{10} \mathrm{OPU} / \mathrm{ml}$ (Ad-GFP-NT3) and $4.33 \times 10^{10} \mathrm{OPU} / \mathrm{ml}$ (Ad-GFP-BDNF). AdGFP-NT3 and Ad-GFP-BDNF were mixed in a $1: 1$ ratio just prior to injection and will hereon be referred to as Ad-NTs. 
Experimental animals and ethics. Male or female adult pigmented Dunkin-Hartley GPs ( $n=5$, average weight $323 \pm 24.5 \mathrm{~g}$ ) were used in this study. Viral administration was performed with the approval of the Office of the Gene Technology Regulator Australia (Licence \#444). National Health and Medical Research Council of Australia and National Institutes of Health (NIH, USA) Guidelines for the Care and Use of Laboratory Animals were observed. The Animal Research Ethics Committee of the Royal Victorian Eye and Ear Hospital approved the care and use of the animals in this study.

Deafening. The hearing status of each GP was assessed prior to deafening by measuring auditory brainstem responses $(\mathrm{ABR})$ to computer-generated click stimuli ${ }^{27}$. For inclusion in the study GPs were required to have normal hearing, which was defined as an $\mathrm{ABR}$ threshold $<43 \mathrm{~dB}$ peak-equivalent sound pressure level. Animals meeting this criterion were deafened under gaseous anaesthesia (Isoflurane; Abbott Laboratories, IL) via intravenous infusion of $100 \mathrm{mg} / \mathrm{kg}$ furosemide (Troy Laboratories, Smithfield, Australia) and subcutaneous $400 \mathrm{mg} / \mathrm{kg}$ kanamycin sulphate (Applichem, Taren Point, Australia) ${ }^{28}$. Deafness was confirmed one week post procedure using ABRs, where animals with a threshold shift of $>50 \mathrm{~dB}$ were considered profoundly deaf.

Cochlea injection of viral sample. Two microliters of Ad-NTs, were unilaterally injected into the lower basal scala media of the cochlea one week post deafening as previously described ${ }^{7,10}$. This approach was selected to specifically target the OC for gene transfection.

Histology. Six months post injection GPs were deeply euthanised and intracardially perfused as previously described ${ }^{7}$. The bullae were removed and the cochleae exposed. Cochleae were decalcified, embedded in OCT and sectioned as previously

described $^{7,29}$. Half of the cochlea was retained for surface preparations as described ${ }^{7,10}$. Standard immunofluorescent protocols were followed using antibodies against heavy chain neurofilament $(1: 200$, NF-H; Merck Millipore, Australia) to stain the SGNs and peripheral fibres, anti-calretinin (1:500, Merck Millipore, Australia) to stain cells within the OC, anti-BDNF (1: 100, Santa Cruz Biotechnology, Santa Cruz, CA) to confirm NT production. AlexaFluor secondary antibodies $(1: 200-1: 500$, Molecular Probes, USA) were used to visualise several antibodies in the same sample and mounted in media containing DAPI. Sections were examined on a Zeiss Axioplan fluorescence microscope (Carl Zeiss, Germany). Cochlear half-turn surface preparations and pre mid-modiolar sections were viewed on a Zeiss Meta confocal microscope.

Data analysis. GFP expression. The level of GFP expression was analysed from three non-consecutive (greater than $72 \mu \mathrm{m}$ apart) mid-modiolar sections from each cochlea. GFP-positive images were thresholded using ImageJ software (NIH, USA) and the pixel density of each image was measured. These measurements were averaged to obtain a single measurement for each animal. Statistical analysis of expression was performed using a one-way ANOVA and presented as mean \pm SEM.

$O C$ degeneration. The extent of OC degeneration was quantified by measuring the area of the OC in the upper basal turn from three non-consecutive cochlear sections (greater than $72 \mu \mathrm{m}$ apart) stained with Mayer's haemotoxylin and Putt's eosin midmodiolar cross section using ImageJ software. The upper basal turn was used for quantification as it had high levels of adenoviral transduction without the confounding effect of the piercing through the basilar membrane to access the scala media, which was present in the lower basal turn.

SGN density. SGN density was analysed blindly from three non-consecutive (greater than $72 \mu \mathrm{m}$ apart) mid-modiolar sections from each cochlea. Density was calculated by counting co-labelled NF-H and DAPI-positive SGN cell bodies within Rosenthal's canal, and then dividing the number of SGNs by the area of Rosenthal's canal using ImageJ software. SGN densities in lower and upper basal, middle and apical turn were averaged to calculate the overall density of each cochlear region. Statistical analyses of SGN density data were performed using a paired student's t-test and presented as mean \pm SEM.

Fibre density within the osseous spiral lamina. Fibre density within the osseous spiral lamina (OSL) was analysed blindly from three non-consecutive (greater than $72 \mu \mathrm{m}$ apart) mid-modiolar haematoxylin and eosin $(\mathrm{H}+\mathrm{E})$ stained sections from each cochlea. This was calculated by inverting the images and tracing the OSL to ascertain the mean grey value within this region, using ImageJ software. Statistical analyses of peripheral fibre density data were performed using a paired t-test and presented as mean \pm SEM

Histology analysis. The chronic tissue response to the surgery and viral inoculation was assessed by measuring the area of tissue response in mid-modiolar cross sections and calculating that as a percentage of the total area of the scala tympani. Tissue response was quantified in $\mathrm{H}+\mathrm{E}$ sections in three non-consecutive cochlear sections (greater than $72 \mu \mathrm{m}$ apart) using a Zeiss Axio Imager M2 microscope and analysed using Image J.

1. LeWitt, P. A. et al. AAV2-GAD gene therapy for advanced Parkinson's disease: a double-blind, sham-surgery controlled, randomised trial. Lancet Neurol 10, 309-319, doi:10.1016/S1474-4422(11)70039-4 (2011).
2. Luo, J. et al. Subthalamic GAD gene therapy in a Parkinson's disease rat model Science 298, 425-429, doi:10.1126/science.1074549 (2002).

3. Ashtari, M. et al. The human visual cortex responds to gene therapy-mediated recovery of retinal function. J Clin Invest 121, 2160-2168, doi:10.1172/JCI57377 (2011).

4. Maguire, A. M. et al. Safety and efficacy of gene transfer for Leber's congenital amaurosis. N Engl J Med 358, 2240-2248, doi:10.1056/NEJMoa0802315 (2008).

5. Akil, O. et al. Restoration of hearing in the VGLUT3 knockout mouse using virally mediated gene therapy. Neuron 75, 283-293, doi:10.1016/j.neuron.2012.05.019 (2012).

6. Izumikawa, M. et al. Auditory hair cell replacement and hearing improvement by Atoh1 gene therapy in deaf mammals. Nat Med 11, 271-276 (2005).

7. Wise, A. K. et al. Effects of localized neurotrophin gene expression on spiral ganglion neuron resprouting in the deafened cochlea. Mol Ther 18, 1111-1122, doi:10.1038/mt.2010.28 (2010).

8. Wise, A. K. et al. The effect of deafness duration on neurotrophin gene therapy for spiral ganglion neuron protection. Hear Res 278, 69-76, doi:10.1016/ j.heares.2011.04.010 (2011).

9. Bankiewicz, K. S. et al. Long-term clinical improvement in MPTP-lesioned primates after gene therapy with AAV-hAADC. Mol Ther 14, 564-570, doi:10.1016/j.ymthe.2006.05.005 (2006).

10. Atkinson, P. J. et al. Neurotrophin Gene Therapy for Sustained Neural Preservation after Deafness. PLoS One 7, e52338, doi:10.1371/ journal.pone.0052338 (2012).

11. Shibata, S. B. et al. Transgenic BDNF induces nerve fiber regrowth into the auditory epithelium in deaf cochleae. Exp Neurol 223, 464-472, doi:10.1016/ j.expneurol.2010.01.011 (2010).

12. Sly, D. J. et al. Deafness alters auditory nerve fibre responses to cochlear implant stimulation. Eur J Neurosci 26, 510-522 (2007).

13. Wise, A. K. et al. Combining cell-based therapies and neural prostheses to promote neural survival. Neurotherapeutics: the journal of the American Society for Experimental NeuroTherapeutics 8, 774-787, doi:10.1007/s13311-011-0070-0 (2011).

14. Taylor, R. R., Jagger, D. J. \& Forge, A. Defining the cellular environment in the organ of Corti following extensive hair cell loss: a basis for future sensory cell replacement in the Cochlea. PLoS One 7, e30577, doi:10.1371/ journal.pone.0030577 (2012).

15. Liu, Y. et al. Protection against aminoglycoside-induced ototoxicity by regulated AAV vector-mediated GDNF gene transfer into the cochlea. Mol Ther 16, 474-480 (2008).

16. Ruan, R. S., Leong, S. K., Mark, I. \& Yeoh, K. H. Effects of BDNF and NT-3 on hair cell survival in guinea pig cochlea damaged by kanamycin treatment. Neuroreport 10, 2067-2071 (1999)

17. Saberan-Djoneidi, D. et al. Molecular dissection of the Schwann cell specific promoter of the PMP22 gene. Gene 248, 223-231 (2000).

18. Nadol, J. B., Jr. Patterns of neural degeneration in the human cochlea and auditory nerve: implications for cochlear implantation. Otolaryngol Head Neck Surg 117 220-228 (1997).

19. Nadol, J. B., Jr., Young, Y. S. \& Glynn, R. J. Survival of spiral ganglion cells in profound sensorineural hearing loss: implications for cochlear implantation. Ann Otol Rhinol Laryngol 98, 411-416. (1989).

20. Otte, J., Schuknecht, H. F. \& Kerr, A. G. Ganglion cell populations in normal and pathological human cochleae. Implications for cochlear implantation. Laryngoscope 88, 1231-1246 (1978).

21. Agterberg, M., Versnel, H., van Dijk, L., de Groot, J. \& Klis, S. Enhanced Survival of Spiral Ganglion Cells After Cessation of Treatment with Brain-Derived Neurotrophic Factor in Deafened Guinea Pigs. J Assoc Res Otolaryngol 10, 355-367 (2009)

22. Gillespie, L. N., Clark, G. M., Bartlett, P. F. \& Marzella, P. L. BDNF-induced survival of auditory neurons in vivo: Cessation of treatment leads to accelerated loss of survival effects. J. Neurosci. Res. 71, 785-790 (2003).

23. Pettingill, L. N., Wise, A. K., Geaney, M. S. \& Shepherd, R. K. Enhanced auditory neuron survival following cell-based BDNF treatment in the deaf guinea pig. PLoS One 6, e18733, doi:10.1371/journal.pone.0018733 (2011).

24. Tan, J. et al. Nanoporous peptide particles for encapsulating and releasing neurotrophic factors in an animal model of neurodegeneration. Adv Mater 24, 3362-3366, doi:10.1002/adma.201200634 (2012).

25. James, D. P., Eastwood, H., Richardson, R. T. \& O'Leary, S. J. Effects of Round Window Dexamethasone on Residual Hearing in a Guinea Pig Model of Cochlear Implantation. Audiol Neurootol 13, 86-96 (2008).

26. Backhouse, S., Coleman, B. \& Shepherd, R. Surgical access to the mammalian cochlea for cell-based therapies. Exp Neurol 214, 193-200 (2008).

27. Shepherd, R. K., Coco, A., Epp, S. B. \& Crook, J. M. Chronic depolarization enhances the trophic effects of brain-derived neurotrophic factor in rescuing auditory neurons following a sensorineural hearing loss. J Comp Neurol 486, 145-158 (2005)

28. Richardson, R. T. et al. Polypyrrole-coated electrodes for the delivery of charge and neurotrophins to cochlear neurons. Biomaterials 30, 2614-2624 (2009).

29. Wise, A. K., Richardson, R., Hardman, J., Clark, G. \& O'Leary, S. Resprouting and survival of guinea pig cochlear neurons in response to the administration of the 
neurotrophins brain-derived neurotrophic factor and neurotrophin-3. J Comp Neurol 487, 147-165 (2005).

\section{Acknowledgments}

The authors would like to acknowledge and thank Assistant professor Clifford Hume from the University of Washington for the adenovirus used in this paper. The authors would also like to acknowledge the funding support from The Garnett Passe and Rodney Williams Memorial Foundation, Action on Hearing Loss, The University of Melbourne Department of Otolaryngology, The Royal Victorian Eye and Ear Hospital, National Health and Medical Research Council (GNT 1024350). The authors would like to acknowledge the support from the State Government of Victoria's Operational Infrastructure Support Program.

\section{Author contributions}

P.J.A. and B.O.F. conducted the experiments; P.J.A., A.K.W., B.A.N., R.T.R. wrote the manuscript, P.J.A., A.K.W., B.A.N., R.T.R. designed experiments, P.J.A., A.K.W., R.T.R. collected and interpreted the data.

\section{Additional information}

Competing financial interests: The authors declare no competing financial interests.

How to cite this article: Atkinson, P.J., Wise, A.K., Flynn, B.O., Nayagam, B.A. \&

Richardson, R.T. Viability of Long-Term Gene Therapy in the Cochlea. Sci. Rep. 4, 4733; DOI:10.1038/srep04733 (2014).

This work is licensed under a Creative Commons Attribution-NonCommercialNoDerivs 3.0 Unported License. The images in this article are included in the article's Creative Commons license, unless indicated otherwise in the image credit; if the image is not included under the Creative Commons license, users will need to obtain permission from the license holder in order to reproduce the image. To view a copy of this license, visit http://creativecommons.org/licenses/by-nc-nd/3.0/ 